\title{
Protein unlocking procedures of formalin-fixed paraffin-embedded tissues: Application to MALDI-TOF Imaging MS investigations
}

\author{
Maurizio Ronci, 2, 3, Elena Bonanno ${ }^{4}$, Alfredo Colantoni ${ }^{4}$, Luisa Pieroni ${ }^{1,2,3}$, \\ Carmine Di Ilio ${ }^{1,2}$, Luigi Giusto Spagnoli ${ }^{4}$, Giorgio Federici, 6, 6, and Andrea Urbani ${ }^{1,2,3}$ \\ ${ }^{1}$ Centro Studi sull'Invecchiamento, Chieti, Italy \\ 2 Dipartimento di Scienze Biomediche, Università "G. D'Annunzio" di Chieti-Pescara, Italy \\ ${ }^{3}$ IRCCS-Fondazione Santa Lucia Centro di Ricerca sul Cervello, Rome, Italy \\ ${ }^{4}$ Dipartimento di Biopatologia Diagnostica per Immagini sez. di Anatomia Patologica, \\ Università di Roma "Tor Vergata", Rome, Italy \\ ${ }^{5}$ Dipartimento di Medicina Interna, Università di Roma "Tor Vergata", Rome, Italy \\ ${ }^{6}$ Dipartimento di Medicina di Laboratorio, Policlinico di Tor Vergata, Rome, Italy \\ ${ }^{7}$ Ospedale Pediatrico Bambin Gesù - IRCCS, Rome, Italy
}

Archival formalin-fixed paraffin-embedded (FFPE) tissues are a powerful tool for examining the clinical course of diseases. These specimens represent an incredible mine of valuable clinical and biological information for proteomic investigation. MALDI-TOF imaging MS (MALDI-IMS) is a protein profiling technique which enables the direct sampling of histological section; however, the quality of molecular data are strongly influenced by the tissue preparation condition. In fact, in previous years most of the studies employing such a technological platform have been conducted using cryo-preserved tissues. We have developed an in vitro approach using "tissue surrogate" samples in order to explore different protein unlocking procedures which might enable a suitable recovery of polypeptides for MS analysis. The developed protocols have been compared both by MALDI-TOF MS and $\mathrm{nLC}-\mathrm{MS}^{\mathrm{E}}$ analysis either on surrogate samples or on FFPE specimen from human breast cancer. The collected evidence has been applied for the preparation of FFPE tissue sections following MALDI-IMS analysis. Our results outline the possibility to obtain valuable peptide mass spectra profiles form FFPE preparations by applying a combined two steps procedure of heat induced antigen retrieval (HIAR) in presence of EDTA and on target trypsin hydrolysis. A multivariate statistical evaluation is presented and discussed according to molecular spatial distributions and tissue morphology.

\section{Keywords:}

Biomarker discovery / FFPE tissue / Imaging / Mass spectrometry
Received: December 10, 2007

Revised: March 5, 2008

Accepted: March 17, 2008
Correspondence: Professor Andrea Urbani, Centro Studi sull'invecchiamento (Ce.S.I.), Centre of Investigation on Aging, Università degli Studi di Chieti e Pescara, Via Colle dell'Ara, Chieti Scalo, 66100 Chieti, Italy

E-mail: a.urbani@unich.it

Fax: +39-0871-541598

Abbreviations: FFPE, formalin-fixed paraffin-embedded; HIAR, heat induced antigen retrieval; MALDI-IMS, MALDI-TOF imaging MS; SA, sinapinic acid

\section{Introduction}

Human tissue removed by surgery both for diagnostic and therapeutic purposes represent the best source to study molecular markers that characterize complex processes such as carcinogenesis. Formalin-fixed paraffinembedded (FFPE) tissues are collected and stored for a long time after the microscopic evaluation and represent a good chance to delineate the proteomic profile of spe- 
cific diseases. The major advantage of FFPE tissues is the vast archives of samples available. Tissue fixation through the formation of protein crosslinks (resulting from the formaldehyde reactivity with side-chain residues, e.g., lysine, asparagines, and glutamine) stabilize the cellular morphology preventing autolytic phenomena and organelles redistribution inside the cells [1, 2]. Nevertheless as a major drawback it renders impossible the application of the routinely used procedures of biochemical protein extraction and the subsequent protein structural analysis $[3,4]$.

Even though evaluations of protein separation studies from frozen tissues has been demonstrated to be more feasible, several separation studies from FFPE sections have been performed and new methods to unlock the formalin-fixed proteins developed. In the field of proteomics investigations Ahram et al. [3] demonstrated that the proteins can be extracted from ethanol-fixed and paraffin-embedded tissue, and resolved by 2-DE separation. Recently Shi et al. [4] obtained a satisfying overlapping between proteins identified from FFPE and frozen tissues and encouraging results are reported also by Prieto et al. [5] and Crockett et al. [6] that identified proteins from FFPE enzymatically digested tissues. However, few studies have been pursuing MALDI-TOF imaging MS (MALDI-IMS), the most challenging proteomics analysis application on FFPE section.

MALDI-IMS is a recently improved technology which combines the high potential of MALDI-TOF MS to identify large size molecules (protein) in biological samples with the opportunity to examine histological sections, and directly correlate the molecular analysis with tissue localization [7-9]. MALDI is a soft ionization technique where molecules are generally embedded in a saturated matrix solution. Sinapinic acid (SA) has been reported as a matrix of choice for the protein analysis both in the case of linear MALDI-TOF MS and in the case of MALDI-IMS [9]. It has a high gas-phase basicity (206 kcal/mol) that is particularly suitable for entire protein's MALDI ionization, given its low tendency in analyte fragmentation [10].

New laser generation has dramatically changed the analytical performances of MALDI-IMS. Common Nd:YAG lasers (solid state) are restricted to limited sample preparation options in proteomics (only dried droplet HCCA preparations work well) [10]. With alternative matrices, such as SA, the sample is exhausted after a few laser shots, whereas the same sample is more durable and results in significantly better spectra quality using a nitrogen laser for desorption/ionization. On the other hand, nitrogen lasers are limited to a shot frequency of $\sim 50 \mathrm{~Hz}$. Smartbeam technologies provides the best performances for MALDI imaging applications due to the combination of the speed of a solid-state laser and the spectra quality of a $\mathrm{N}_{2}$ laser thus resulting in higher protein resolution and a three-fold increase in sensitivity [10].
Moreover, the higher pulse frequencies enable almost a ten-fold decrease in the acquisition time to complete MALDI-IMS dataset, therefore allowing to afford clinically relevant sample size. These novel features give to this technology, big potentiality in the field of biomarkers discovery with diagnostic purposes [11]. The identification of biomarkers directly from tissue section would be of extreme importance for the diagnosis of diseases, especially tumors. This is the major aim for the development of MALDI-IMS technology, whose potential is even stronger than MALDI-MS considering that it gives the possibility to provide profiles and 2-D ion density maps of molecules directly from raw tissue sections, showing the relative abundance and spatial distribution of molecules [11]. Recently, Schwartz et al. [12] obtained good results in the prognosis of brain tumor patient by MALDI-IMS carried out on fresh frozen tissues. Furthermore, Lemaire et al. [13] showed that a combination of in situ extraction with in situ microdigestion performed on FFPE tissues allows the identification of proteins by nanoLC-nanoESI and MALDI imaging.

In the present work, we pursued a methodological improvement of MALDI-IMS aimed to develop protein unlocking procedures which might enable a suitable recovery of polypeptides for MS analysis of FFPE specimens. We explored different procedures using "tissue surrogate" samples, as proposed by Fowler et al. [14]. The collected evidences have been benchmarked on FFPE tissue section and the results discussed in terms of protein coverage and structural information (Fig. 1). This study may represent an initial step toward the application of MALDI-IMS in the clinical screening of FFPE histological samples.

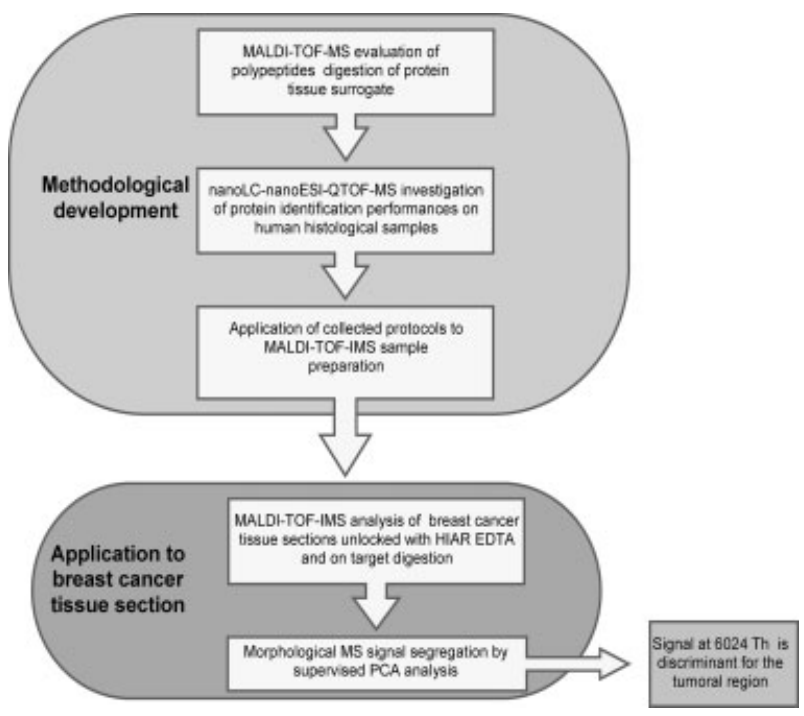

Figure 1. Schematic diagram of the pursued experimental approach, from the development of an IMS strategy to recover polypeptides from FFPE conserved tissues, to its application on breast cancer samples. 


\section{Materials and methods}

\subsection{Chemicals}

All chemicals were of the highest purity obtainable. Water, ACN, and ethanol HPLC-grade, TFA, and formic acid (FA) were from Romil (Cambridge, UK). Ammonium carbonate $\left(\mathrm{NH}_{4} \mathrm{CO}_{3}\right)$, iodoacetamide (IAA), DTT, 3,5-dimethoxy-4hydroxy-cinnamic acid (SA), Tris, urea, and $\left[\mathrm{Glu}^{1}\right]$-fibrinopeptide were purchased from Sigma (St. Louis, MO, USA). Sequencing grade modified trypsin was purchased from Promega (Madison, WI, USA). Peptide standard mix and protein standard mix for MALDI-TOF calibration were from Bruker-Daltonics (Germany). All buffers and reagents were used as supplied from the manufacturer and prepared freshly in Milli-Q grade water. Agarose and acrylamide/bisacrylamide $40 \%$ stock, used for tissue surrogate preparation, were both from Sigma.

\subsection{Sample preparation}

In order to critically assess the different sample preparations and unlocking procedures we have applied a systematic approach from albumin tissue surrogate up to breast cancer thin sections. The overall experimental strategy is reported in Fig. 1.

Tissue surrogates were obtained embedding 10\% BSA, dialyzed against Milli-Q water by means of a 6-8000 MWCO membrane both in agarose gels ( $3 \%$ in Milli-Q water) and in acrylamide gels (10\% in Tris-glycine buffer).

Gels were cut into small pieces (approximately $0.5 \mathrm{~cm}^{3}$ each) and either fixed in $10 \%$ buffered formalin for $24 \mathrm{~h}$ at room temperature, or stored at $4^{\circ} \mathrm{C}$ as control groups.

Tissue samples were prepared from human breast cancer specimens removed by surgery in accordance with the standard local therapeutic protocol. The study was conducted in accordance with the principles of the Helsinki Declaration (World Medical Association, 1997).

Unfixed samples were snap-frozen in isopenthane previously chilled in liquid nitrogen, then stored at $-80^{\circ} \mathrm{C}$ until analyzed. Frozen sections ( $6 \mu \mathrm{m}$ thick) were cut with a Leyca CM 1850 cryostat at $-25^{\circ} \mathrm{C}$. Consecutive sections $(0,100$, and $200 \mu \mathrm{m}$ in depth) were either directly transferred onto a metal ground stainless-steel MALDI target plate, or picked up on glass slides and stained with $H \& E$.

FFPE samples were fixed in $10 \%$ buffered formalin for $24 \mathrm{~h}$, at room temperature, dehydrated with ethylicalcohol and paraffin-embedded. Six micron thick sections were cut and transferred onto a metal ground stainlesssteel MALDI target plate into three separate lines. The plate was kept in a $60^{\circ} \mathrm{C}$ oven overnight to favor section adhesion. Different unlocking procedures have been tested as described in the Section 3 in combination with or without the heat induced antigen retrieval (HIAR) protocols.
For HIAR EDTA and HIAR trypsin/EDTA unlocking procedures, tissues were incubated with $0.1 \mathrm{M}$ EDTA $\mathrm{pH} 8.4$ or $0.1 \mathrm{M}$ EDTA pH 8.4 plus $3 \%$ trypsin for $30 \mathrm{~min}$ in $98^{\circ} \mathrm{C}$ bath, respectively. In $37^{\circ} \mathrm{C}$ trypsin unlocking procedure the incubation with $3 \%$ trypsin was performed in PBS for $15 \mathrm{~min}$ at $37^{\circ} \mathrm{C}$.

\subsection{MALDI-TOF imaging-MS analysis}

Tissue sections were washed with $500 \mu \mathrm{L} 70 \% \mathrm{EtOH}$ directly on the target plate. Only one replicate per section was subject to further tryptic digestion before the spectrometric analysis as follows: after air drying, $25 \mu \mathrm{L}$ of $50 \mathrm{mM}$ ammonium carbonate buffer solution, containing sequence grade trypsin at $10 \mathrm{ng} / \mu \mathrm{L}$, was applied taking care to cover all tissue surface and to drain off the solution in excess. Tissues were incubated at room temperature until dry and then another cycle of digestion was performed (typically $30 \mathrm{~min}$ ). Matrix coating was performed for both digested and nondigested sample by uniformly spraying a $10 \mathrm{~g} / \mathrm{L}$ solution of SA, dissolved in $80 \% \mathrm{ACN}$, and $0.1 \%$ TFA by means of a chromatographic TLC sprayer. Typically 15-20 cycles of successive spray deposition were done. All data were collected on an UltraFlex III MALDI-TOF/TOF mass spectrometer (Bruker-Daltonics) equipped with a smartbeam ${ }^{\text {TM }}$ laser operating at $100 \mathrm{~Hz}$.

FlexImaging 1.0 (Bruker-Daltonics) was used to set up and control the acquisition. Spatial resolution was set to $300 \mu \mathrm{m}$. For each section the acquisition was performed in positive linear mode and in positive reflectron mode. All linear acquisitions, in the range of $2-20 \mathrm{kDa}$, were obtained with voltages of 25 and $23.7 \mathrm{kV}$ for the first and second ion extraction stages, respectively and $6.5 \mathrm{kV}$ for the lens, and a laser power attenuator of $60 \%$. All reflectron acquisitions were performed in the range of 800-7000 Da with voltages of 25 and $21.7 \mathrm{kV}$ for the first and second ion extraction stages, respectively and $9 \mathrm{kV}$ for the lens, $26.3 \mathrm{kV}$ for reflector 1 , $13.8 \mathrm{kV}$ for reflector 2 , and a laser power of $40 \%$. Two hundred fifty shots per spectrum were accumulated for the linear mode and 500 for the reflectron mode. Quadratic external calibration of TOF was performed on average mass of ACTH (clip 18-39) $[\mathrm{M}+\mathrm{H}]^{+} \quad(m / z=2466.681)$, somatostatin $[\mathrm{M}+\mathrm{H}]^{+} \quad(m / z=3149.574), \quad$ insulin $\quad[\mathrm{M}+\mathrm{H}]^{+} \quad(\mathrm{m} / z$ =5734.52), cytochrome $C[\mathrm{M}+2 \mathrm{H}]^{2+}(m / z=6181.05)$, myoglobin $[\mathrm{M}+2 \mathrm{H}]^{2+} \quad(m / z=8476.66)$, ubiquitin I $[\mathrm{M}+\mathrm{H}]^{+} \quad(m / z=8565.76)$, cytochrome $C[\mathrm{M}+\mathrm{H}]^{+}(\mathrm{m} / z$ $=12$ 360.97), and myoglobin $[\mathrm{M}+\mathrm{H}]^{+}(\mathrm{m} / z=16$ 952.31). Standard signals in the range of $2-17 \mathrm{kDa}$ were used to calibrate the linear mode acquisition while signals in the range of 1-6 kDa were used for the reflectron mode. Spatial images were directly reconstructed by the software. ClinProTools 2.1 (Bruker-Daltonics) was used to run principal component analysis (PCA) and classification analysis on the acquired datasets. 


\subsection{Protein identification by nUPLC-MSE}

To perform a nUPLC-MSE analysis, samples were trypsin digested as follows: each tissue sample section (breast cancer tissue) was added to $25 \mu \mathrm{L} 6 \mathrm{M}$ urea dissolved in $100 \mathrm{mM}$ Tris $\mathrm{pH} 7.8$.

To reduce and alkylate proteins, $2.5 \mu \mathrm{L}$ DTT $(100 \mathrm{mM})$ $\left(1 \mathrm{~h}\right.$ at $\left.37^{\circ} \mathrm{C}\right)$ and $3 \mu \mathrm{L}$ iodoacetamide $(200 \mathrm{mM})(1 \mathrm{~h}$ at $\mathrm{RT}$ in dark) were added subsequently.

Proteins were digested with $1 \mu \mathrm{L}$ of $0.5 \mu \mathrm{g} / \mu \mathrm{L}$ sequencing grade trypsin at $37^{\circ} \mathrm{C}$ overnight. To stop the reaction $1 \mu \mathrm{L}$ of $10 \% \mathrm{v} / \mathrm{v}$ TFA was added. A $2 \mu \mathrm{L}$ aliquot of the digested peptide was loaded on a column for separation.

The chromatographic system was a nano-ACQUITY UPLC $^{\text {TM }}$ System (Waters). Peptides were trapped on a $5 \mu \mathrm{m}$ Symmetry $\mathrm{C}_{18}$ trapping column, $180 \mu \mathrm{m} \times 20 \mathrm{~mm}$ (Waters) and washed for $10 \mathrm{~min}$ at a flow rate of $5 \mu \mathrm{L}$ / min with mobile phase A $(0.1 \%$ FA). Peptides were then eluted and separated using a $200 \mathrm{~min}$ RP gradient at $300 \mathrm{~nL} / \mathrm{min}$ (3-40\% ACN over $120 \mathrm{~min})$ on a $1.7 \mu \mathrm{m}$ BEH $130 \quad C_{18}$ NanoEase $^{\text {TM }} 75 \mu \mathrm{m} \times 25 \mathrm{~cm}$ nanoscale LC column (Waters). The column temperature was set to $50^{\circ} \mathrm{C}$.

Lock mass ([Glu1]-fibrinopeptide B, $250 \mathrm{fmol} / \mu \mathrm{L}$ ) was constantly infused by the NanoAcquity auxiliary pump at a constant flow rate of $250 \mathrm{~nL} / \mathrm{min}$. The Q-Tof Premier ${ }^{\mathrm{TM}}$ mass spectrometer (Waters) was programmed to switch between low $(4 \mathrm{eV})$ and high (15-40 eV) energies in the collision cell with a scan time of $1.5 \mathrm{~s}$ per function over a mass range of 50-1990 Th.

Continuum LC-MS data were processed and searched using ProteinLynx GlobalServer v2.3 (Waters). The software exploits the embedded ion accounting algorithm and a search limited to a human database to identify proteins. Both tolerances for parent and fragment ions were set to auto-ppm (optimum chosen by the software), one missed cleavage allowed and cysteine carbamidomethylation and oxidation of methionine as modifications. The minimum to get a positive hit was three and the fragment ions matched per peptide, seven fragment ions matched per protein, and two peptides matched per protein.

\section{Results}

\subsection{Tissue surrogates}

In order to explore protein unlocking procedures which might enable a suitable recovery of polypeptides for MS analysis, we have developed an in vitro approach using "tissue surrogate" samples.

Albumin embedded in agarose and acrylamide has been treated according to standard FFPE procedures available for tissue fixation and subsequently trypsin digested to get a mass fingerprint (Fig. 1).
Unfixed tissues have been analyzed in comparison with FFPE sample either subjected or not to heat associated EDTA unlocking procedure.

As expected the total number of signals obtained from the analysis of the FFPE samples was slightly lower than that obtained from unfixed surrogate. More interestingly, the total number of signals obtained by FFPE HIAR EDTA unlocked sample was the highest. This finding is in agreement with the possible formation of new crosslinks among peptides and formaldehyde [15] and suggests also that the unlocking treatment allows proteins to be more accessible to trypsin proteolytic activity (Fig. 2A).

To strengthen the efficacy of EDTA unlocking procedure, in Fig. 2B, we show that the number of albumin specific spectra obtained by FFPE EDTA unlocked samples is comparable with that obtained by unfixed samples. In order to evaluate the number of crosslinked peptides for each condition, heterobifunctional methylene crosslinking has been added as optional modification to the theoretical digest of albumin by means of GPMAW software. The mass list generated has been compared with the experimental one (50 ppm tolerance). The unfixed surrogate presents 2-4 crosslinked peptides matching, probably due to a random event. A comparable number of crosslinked peptides result for FFPE untreated surrogates, explained by the limited accessibility of proteins. An average of 11-15 crosslinked peptides match for HIAR EDTA unlocked surrogates, probably due to a restored accessibility of proteins (Fig. 2C).

In Figs. 2D and $\mathrm{E}$ the MASCOT score and the sequence coverage resulting from the database search are shown. Values measured for these parameters are comparable both for unfixed surrogates and HIAR EDTA unlocked surrogates.

In order to properly assess the reproducibility of these procedures to real FFPE histological samples we have further investigated these protocols on actual human breast cancer specimens.

\subsection{Breast cancer shotgun proteomics investigation}

The developed protocols have been evaluated by a shotgun proteomics approach on specimen from human breast cancers to evaluate the difference between FFPE and freshly frozen tissues.

Such an investigation has taken advantage from a novel isotope free technology employing ultra performance liquid chromatographic separation coupled to high resolution parallelized MS analysis $\left(\mathrm{MS}^{\mathrm{E}}\right)$.

In Fig. 3A we report chromatographic profiles for the $\mathrm{MS}^{\mathrm{E}}$ acquisition of breast cancer tissue sample sections subjected to different unlocking procedures versus unfixed samples.

For FFPE untreated sample we recorded a chromatographic profile with only few peaks following the base peak ion count signals. In all other conditions, chromatograms present a similar profile with a higher number of peaks. The first part of the chromatographic separation is very popu- 
Agarose gel Acrylamide gel
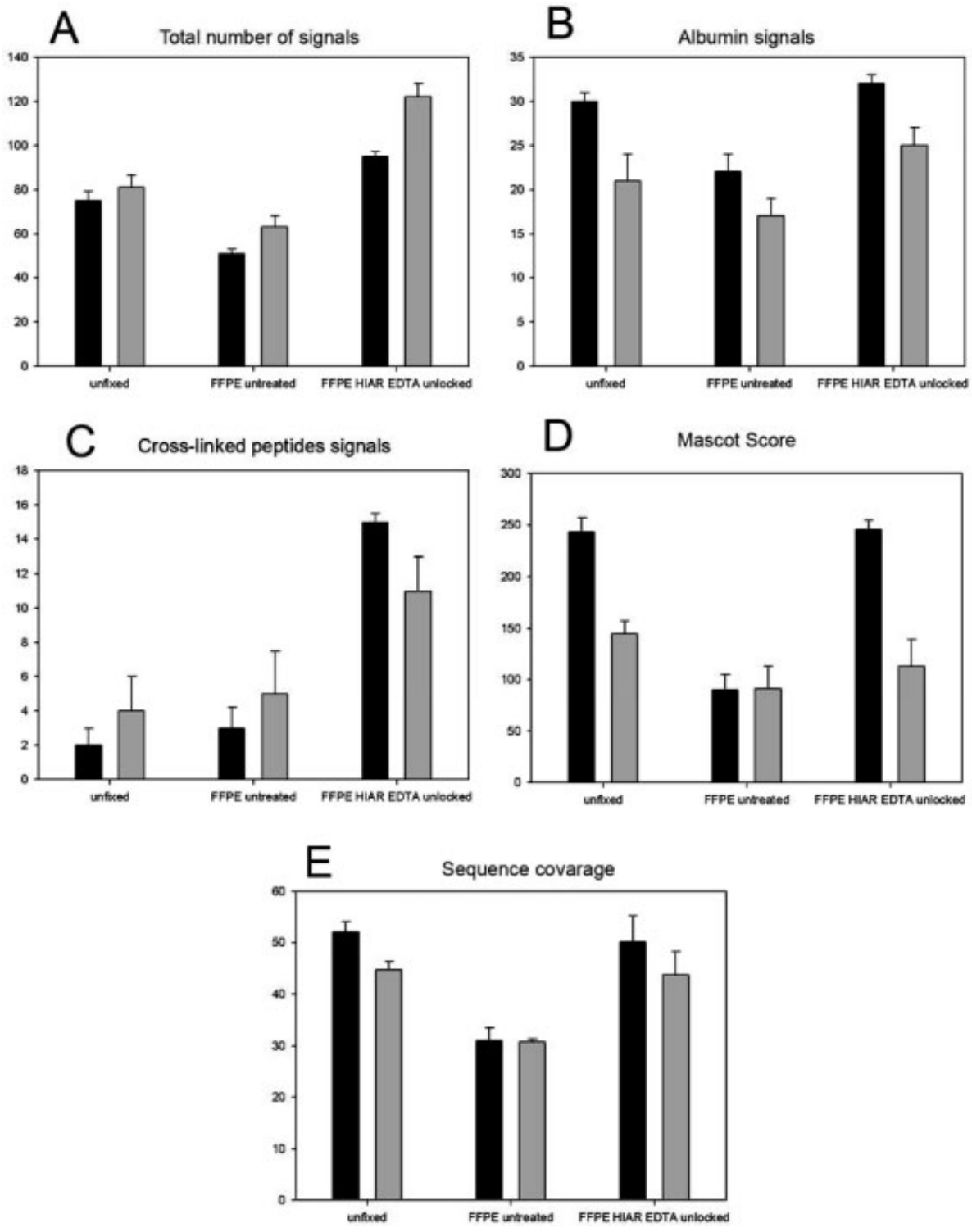

Figure 2. Typical parameters are reported for protein identification by PMF resulting from the MALDI-TOF MS acquisition of digested albumin in tissue surrogates. All formalin-fixed samples are more complex than the unfixed (A: total number of peptides; B: number of matched peptides; C: number of signals of crosslinked peptides); the MASCOT score (D) and the sequence coverage (E) are also higher for the unfixed sample. Formalin fixing without any unblocking treatment result in the lower values for the parameters taken into account while EDTA unblocking treatment results in values comparable with the unfixed. lated. This is typical for such a kind of chromatographic set up. In fact, due to the characteristic $\log P$ value of peptides their $\mathrm{C}_{18}$ column elution profile is in a range between 5 and $30 \%$ ACN. Nevertheless, some differences are visible in the identification performances.

As expected, fresh frozen breast cancer sample tissue turns out to give the highest number of protein identified upon trypsin digestion (137 identified proteins). The same tissue but FFPE stored allow the identification of only eight proteins while HIAR EDTA unlocking treatment, $37^{\circ} \mathrm{C}$ trypsin unlocking treatment, and HIAR trypsin/EDTA unlocking treatment allows the identification of 70,22 , and 59 proteins, respectively (Fig. 3B). A complete list of identified proteins with their identification figures is reported in Table $1 \mathrm{~S}-5 \mathrm{~S}$ of Supporting Information.

The protein quantification performance criteria, as demonstrated by the measured average sequence coverage and average Protein Lynx Global Server (PLGS) score, are not particularly different among the three different unlocking treatments (Figs. 3C and D). In Fig. 3E the number of identified protein in every experimental condition is plotted against the PLGS score value. Given that in our first set of experiment, HIAR trypsin/EDTA unlocking procedure and HIAR EDTA unlocking procedure on FFPE treated sample 


\section{A}
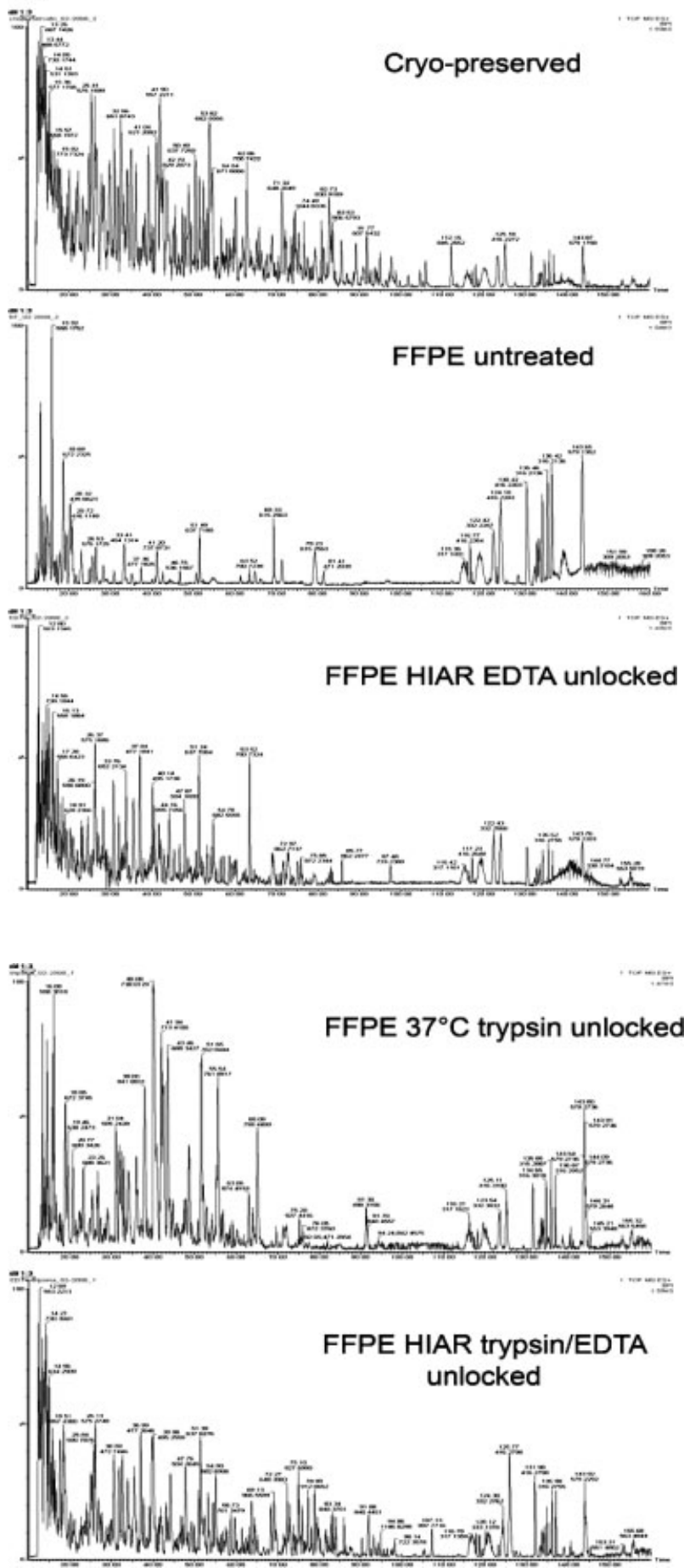

Figure 3. (A) Chromatographic profiles for the MSE acquisition of breast cancer tissue sample sections subject to different unlocking procedures. (B) The histogram graph of identified proteins, (C) the average ProteinLynx Global Server score, (D) and the average sequence coverage for each tested condition. The highest number of identified proteins results for the cryopreserved tissue but it is still good for the HIAR trypsin/EDTA and HIAR EDTA unlocking treatments.
B Number of identified proteins

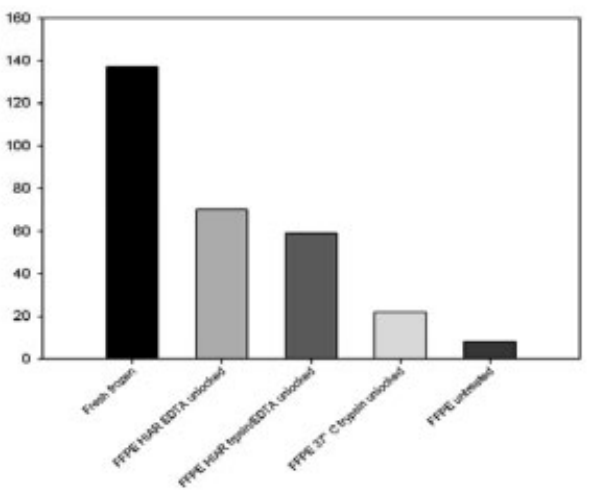

C PLGS score

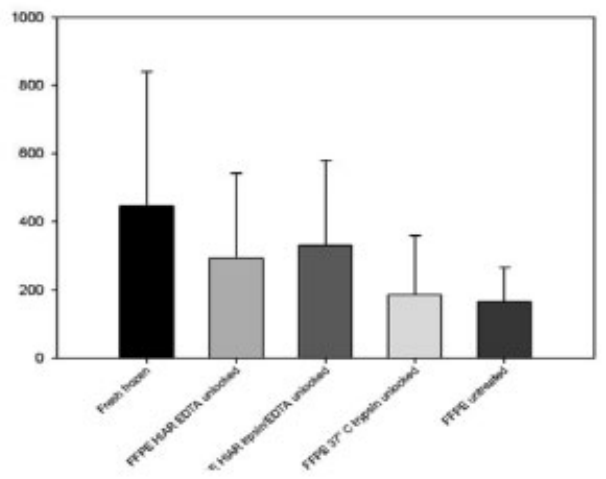

D Average sequence coverage

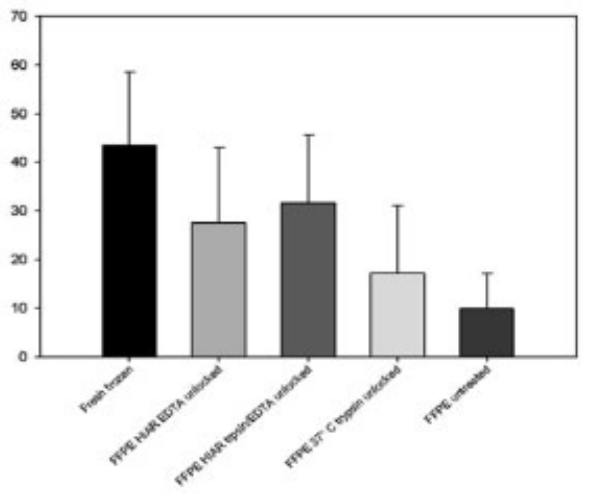

E

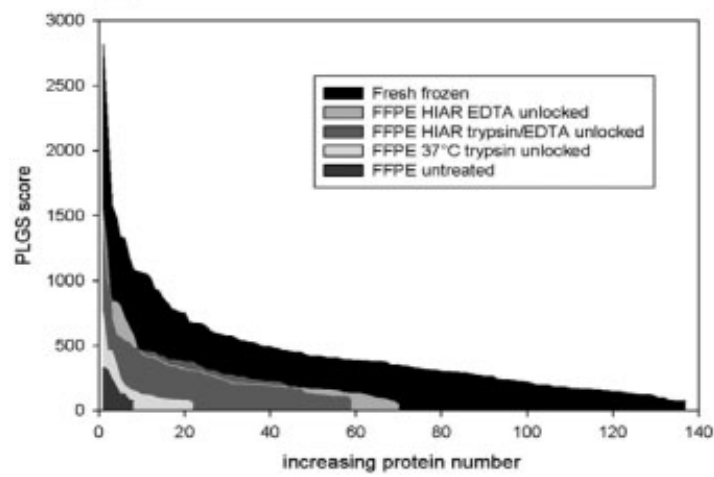


allows a good recovery both in terms of protein accessibility and peptide integrity, we proceed with the implementation of a protocol for MALDI-IMS experiments applying this protocol. These results outline the possibility to obtain peptide mass spectra qualities comparable between the fresh frozen and FFPE tissue preparation enabling direct peptide sequencing experiments.

\subsection{MALDI-IMS on FFPE section}

We initially addressed the implementation of "on-target" trypsin digestion on the histological samples, in order to achieve exhaustive proteolysis. On these histological sections trypsin digestion has been achieved by direct deposition on the MALDI target surface at room temperature. In Figs. 4A and $\mathrm{B}$ we report the average linear mode MALDI-TOF MS spectra of cryo-preserved tissues not digested versus "on target" digested. In the mass spectrum obtained from digested fresh frozen sections the signals are much concentrated in the low mass range, in agreement with the production of small peptides due to trypsin digestion.

Subsequently, we focused our effort on the evaluation of unlocking treatment in combination with on-target trypsin digestion to keep the morphological localization still viable.

FFPE and FFPE unlocked from the same breast cancer samples have been analyzed. All not digested FFPE samples either treated or untreated show poor spectra qualities (data not shown), which is restored only after on-tissue trypsin digestion (Figs. 4C and D). Data acquisitions highlight good quality performance features such as resolution, $\mathrm{S} / \mathrm{N}$ ratio, and total number of signals.

Unfortunately, tissue architecture is only retained when HIAR EDTA unlocking procedure is applied (Fig. 5A). Immunohistochemistry experiments show a diffuse reaction with aspecific stain when $37^{\circ} \mathrm{C}$ trypsin unlocking procedure is applied (Fig. 5B), confirmed by MALDI-IMS analysis (Figs. 5D and E). MALDI-IMS analysis has not been performed for HIAR trypsin/EDTA unlocking procedure because the tissue was damaged from this strong treatment at $98^{\circ} \mathrm{C}$. In fact, we could observe loss of spatial localization also in immunohistochemistry experiments (Fig. 5C) on the small patch of tissue still viable.

In Fig. 6A is reported the optical image of the investigated section, colored according to the classical H\&E method. Different cytological regions are clearly evident: adipose tissue (the white one), connective tissue (the pink one) invaded by tumoral tissue (the dark blue one) and edema tissue (the light pink to white one). In Figs. 6B and $C$ we show the localization of signals at $m / z=6024$ and $m / z=3447$ on HIAR EDTA unlocked FFPE breast cancer sections. Signal 6024 is more abundant in the tumoral tissue (the dark blue area in H\&E staining) and signal 3447 has a specific localization in the edematous area. Interestingly, these two discriminant signals do not show a proper localization when the trypsin $37^{\circ} \mathrm{C}$ unlocking treatment is
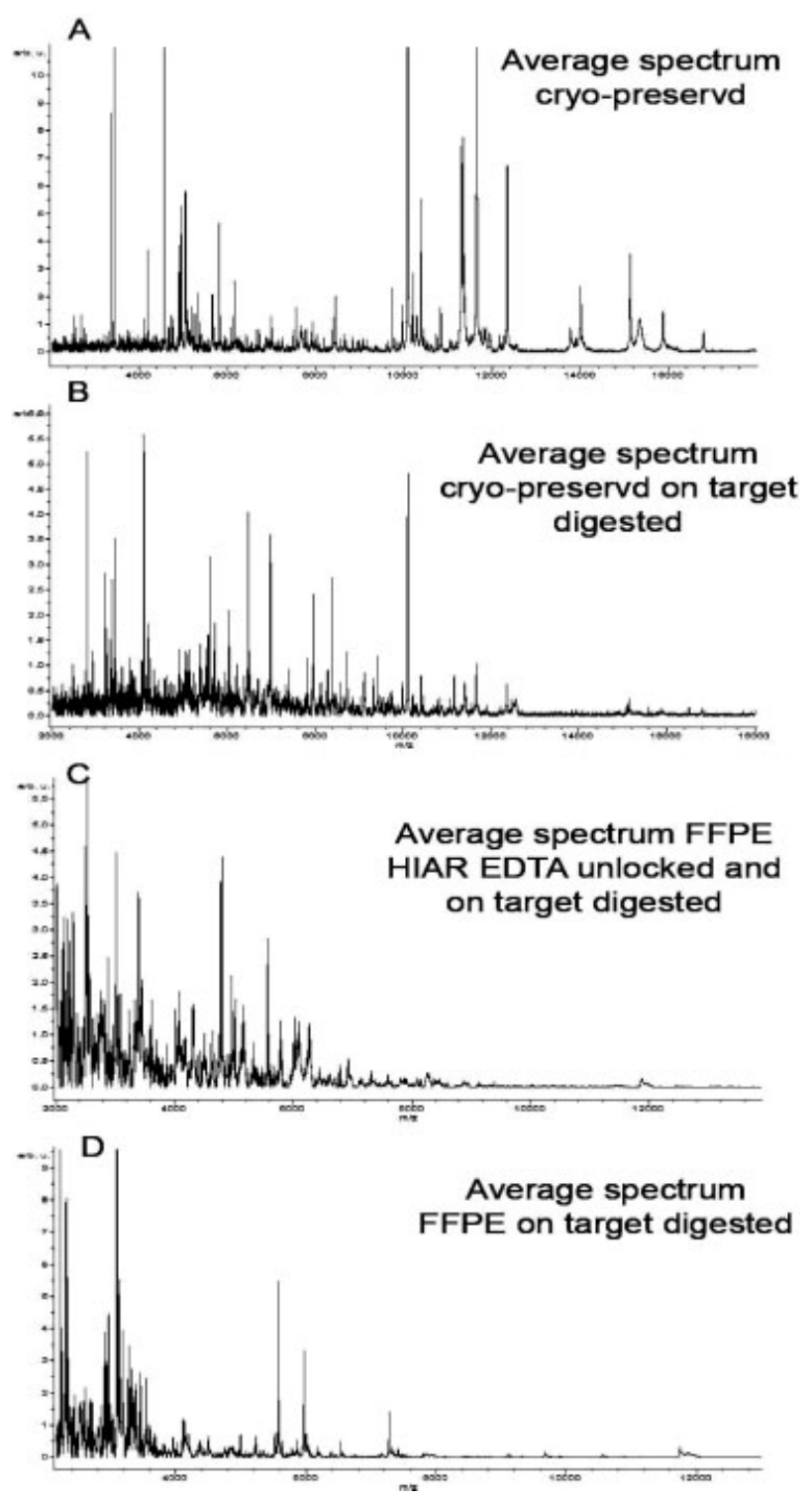

Figure 4. The average spectra for the MALDI-IMS acquisition on breast cancer tissue sections are shown. On comparing the spectra in (A) and (B), the shift of signals toward the low mass range due to ontarget trypsin digestion is evident. FFPE samples both untreated (D) and HIAR EDTA unlocked (C) present a lower number of signals.

employed (Figs. 5D and E), thus confirming the better performances of the HIAR EDTA treatment coupled to on-plate trypsin digestion.

\subsection{Supervised multivariate spectra analysis}

The collected evidences highlight the possibility to employ unlocking procedures for the preparation of FFPE tissue sections to submit to MALDI-IMS analysis. In order to evaluate the quality of tissue spatial features based on MALDIIMS data we have applied a multivariate statistical analysis. 


\section{Immunostaining after unlocking treatments}
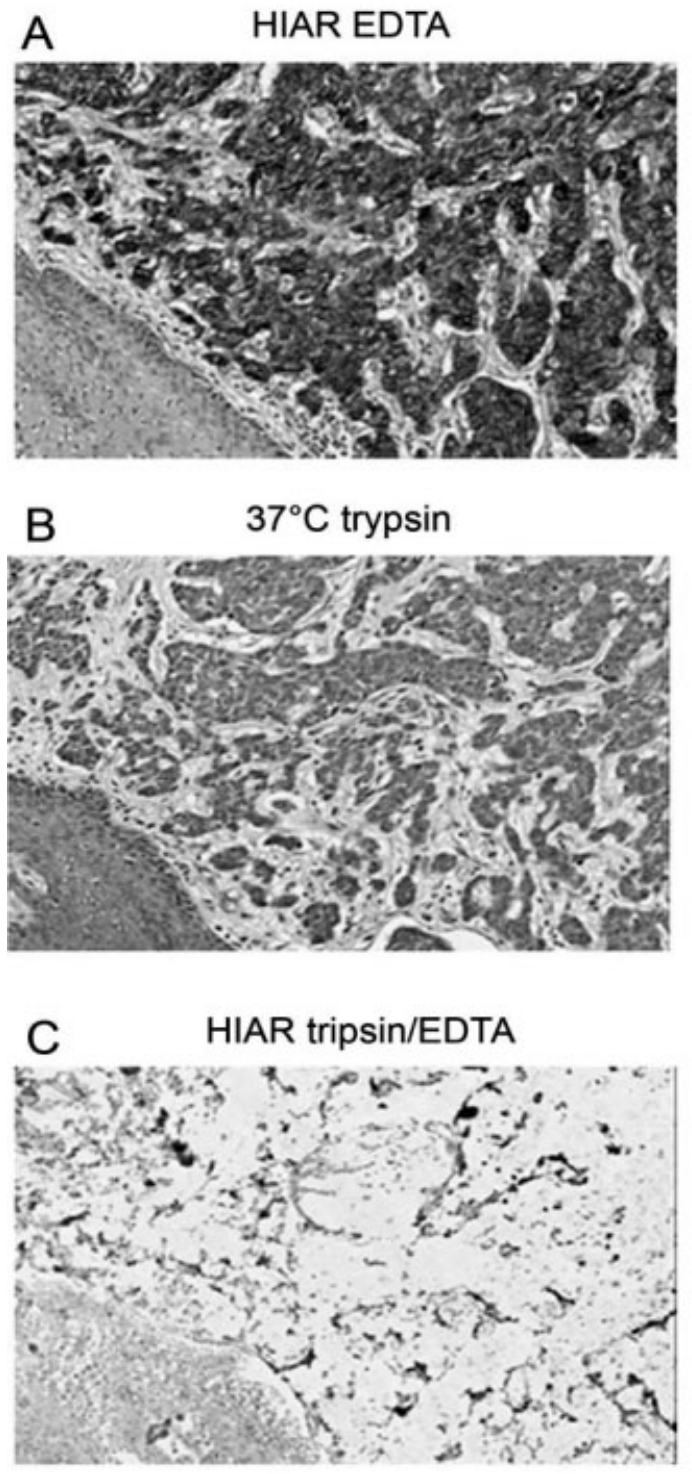

\section{Ionic intensity for signals at 3447 and 6024 after $37^{\circ} \mathrm{C}$ trypsin unlocking treatment}
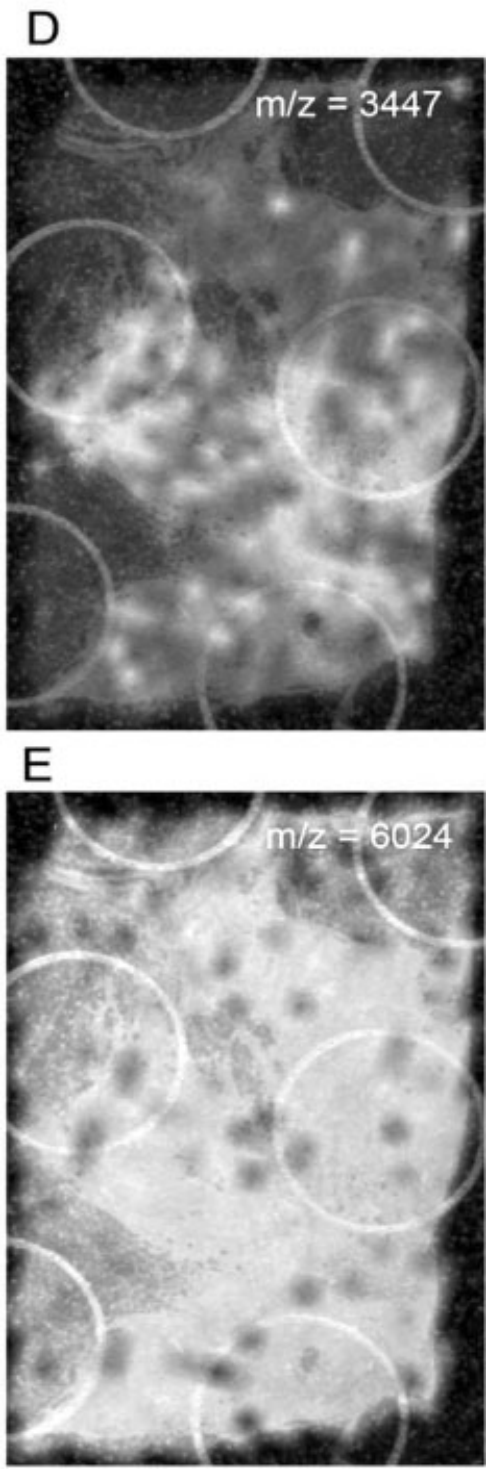

Figure 5. Immunohistochemistry for C-erb-B2 on breast cancer tissue sections after different unlocking procedures. (A) HIAR EDTA shows an intense diffuse positive reaction on the cytoplasmic membrane, morphology is well preserved. (B) $37^{\circ} \mathrm{C}$ trypsin treatment shows a diffuse reaction with aspecific stain in the background, tissue architecture and morphology are preserved. (C) Tissue architecture and morphology are lost when HIAR trypsin/EDTA treatment is applied and no reaction is observed. The ionic image of signals at (D) 3447 and (E) 6024, whose result is not well-spatially localized after $37^{\circ} \mathrm{C}$ trypsin unlocking treatment.
Figure 7A shows an optical image of the investigated section colored according to the classical H\&E method. Given their intrinsic invasive features, tumors infiltrate inside connective tissues making difficult to definitely recognize the two kinds of tissues. To perform a PCA, MS spectra collected from FFPE tissue MALDI-IMS session have been clustered into seven groups, depending on the histological region they belong to (adipose tissue 1, 2, and 3, tumoral region 1 and 2, edema region and connective tissue) and exported to Clinprotools software (Bruker-Daltonics) for the statistical analysis. Adipose tissue 1, tumoral region 2, and edema region were chosen as reference area (Fig. 7B).
They have been first subjected to supervised PCA and then used to generate a classification model. PCA results are shown in Fig. 8A the clustering of residues are mostly distributed in PC1 vs. PC2 and PC1 vs. PC3 graphs. In fact from the graph is evident that more than $50 \%$ of variance is explained by the first PC (Fig. 8B). Such a distribution demonstrates a differential MS profile in the three histological regions analyzed on this section. In order to develop an integrated classification model the set of MS data were subjected to differential peak identification employing a genetic algorithm. Fifteen signals were chosen by the software and identified as differential. The generated model presents a 


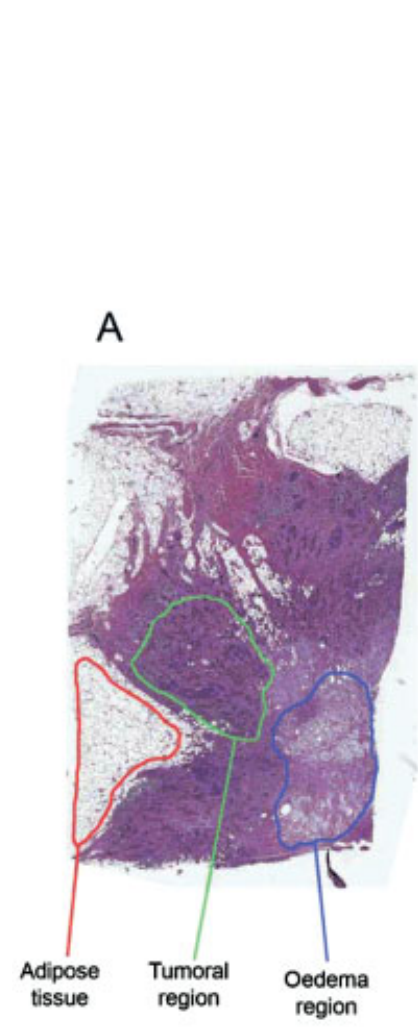

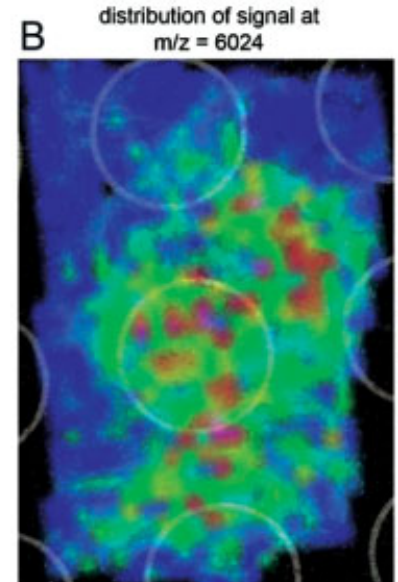
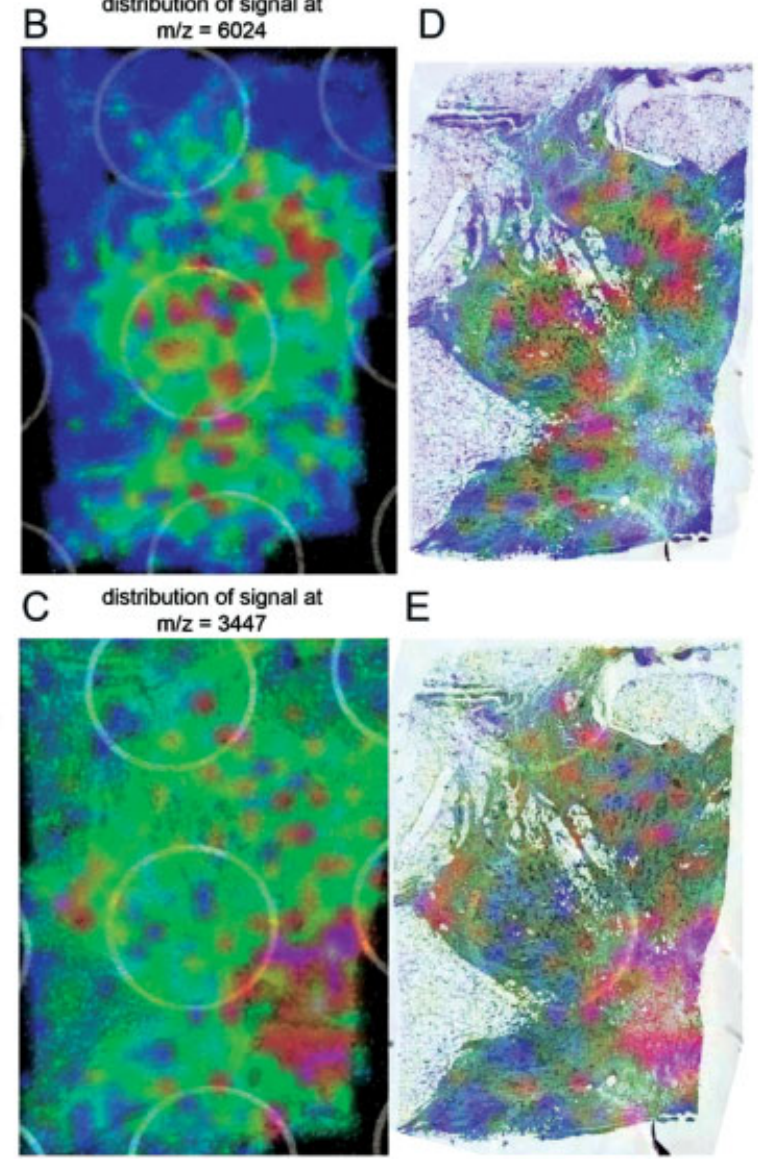

$\mathrm{E}$

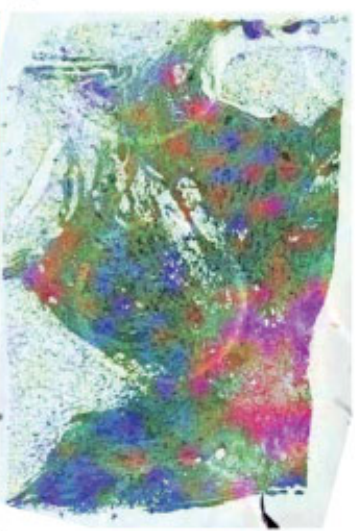

Figure 6. (A) Optical image of the investigated FFPE section colored according to the classical H\&E method; three cytological different regions are highlighted: adipose tissue, tumoral region, and edematous tissue. (B) MALDI-MS protein ion image of a specific signal at $m / z=6024$ of tumoral region. (C) MALDIMS protein ion image of a specific signal at $m / z=3447$ of edema region. In (D) and (E) MALDI-MS protein ion images are superimposed and down mixed with the corresponding optical image to show ontissue localization.

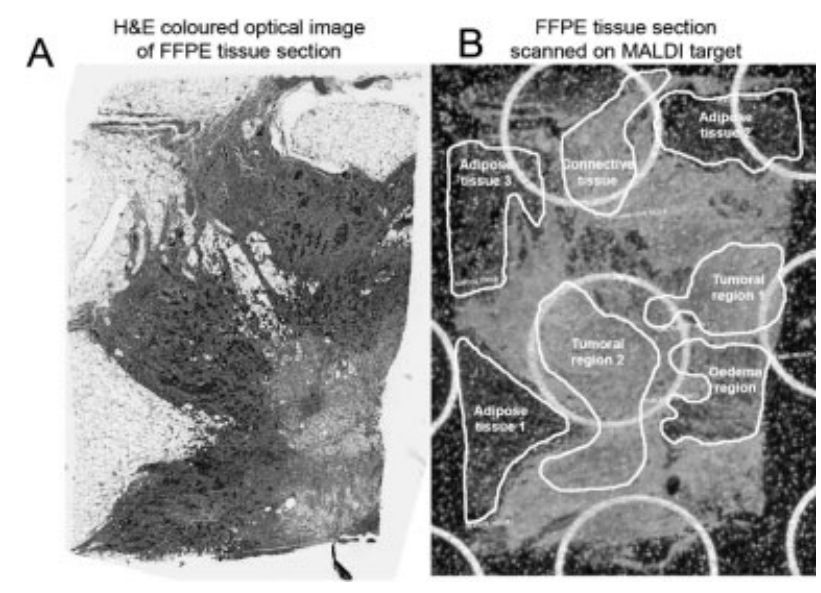

Figure 7. Optical image of the investigated FFPE section colored according to the classical H\&E method (A) compared with the ontarget scanned image (B). Areas chosen for statistical data analysis are highlighted.

recognition capability of $96.0 \%$ and a crossvalidation percentage of 81.21. In Fig. 9A are reported superimposed average spectra (red = adipose tissue; blue = connective tissue; green $=$ tumoral region) for the three classes and the corre- sponding gel view representation. Signals that present the highest variance inside the datasets are highlighted: 3447 and 6024 .

An external validation of the model was executed taking spectra belonging to the regions that were left out from the generation of model and let the model classify them. In Fig. 9B the results of the classification model are reported. Cross, square, and diamond symbols represent adipose tissue, connective tissue, and tumoral region, respectively. Black plume in each panel represent the predictions on external datasets of tumoral region 1 , connective tissue, and adipose tissues 1 and 2, respectively.

The model is able to correctly predict and classify spectra groups.

\section{Discussion}

In this study we described a methodological investigation in the development of unlocking protocols in order to allow MALDI-IMS experiments on FFPE tissue sections. By employing different procedures using "tissue surrogate" samples, as proposed by Fowler et al. [14], and actual human 


\section{A Supervised PCA analysis results}
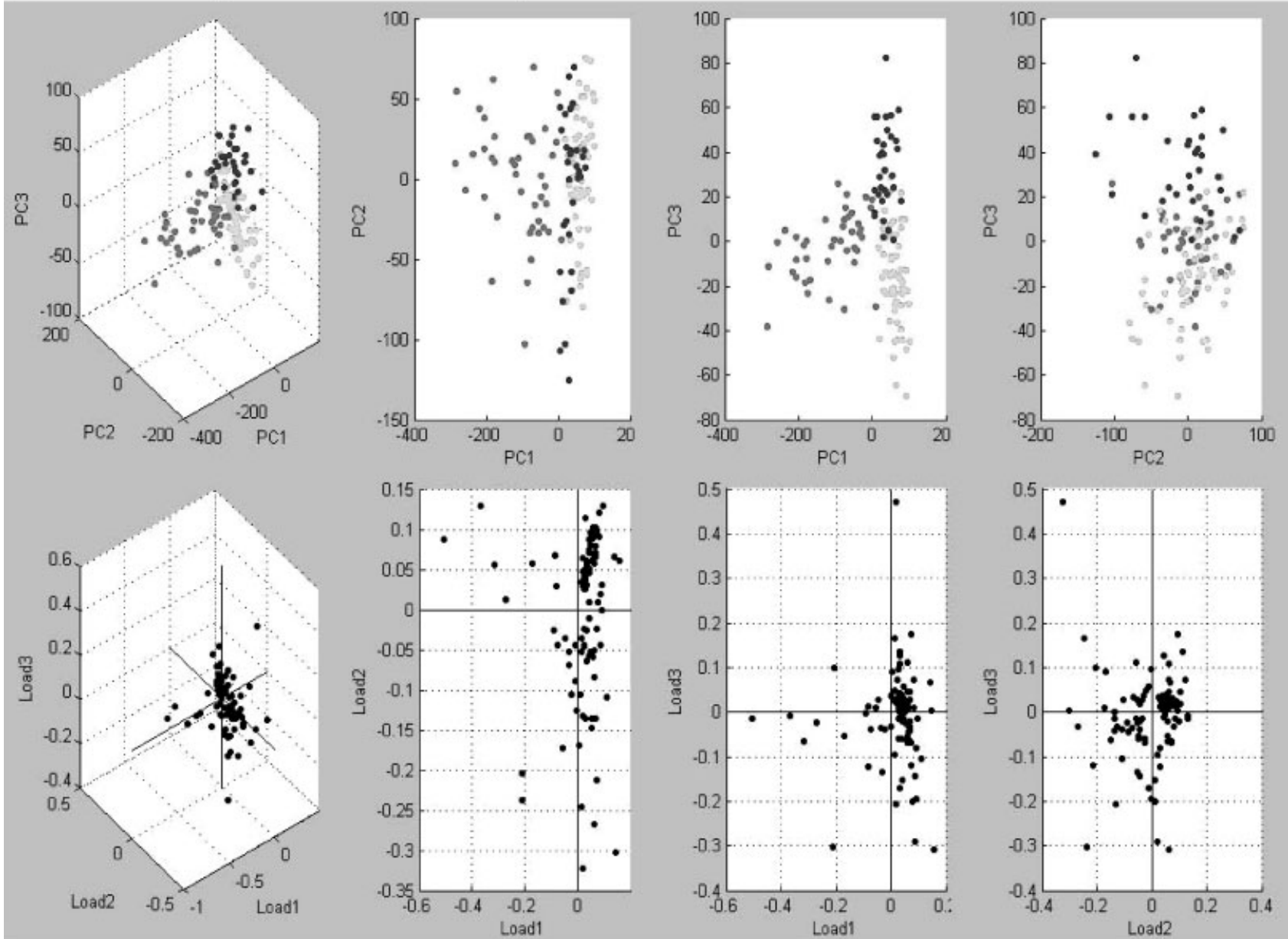

\section{B Explained variance}

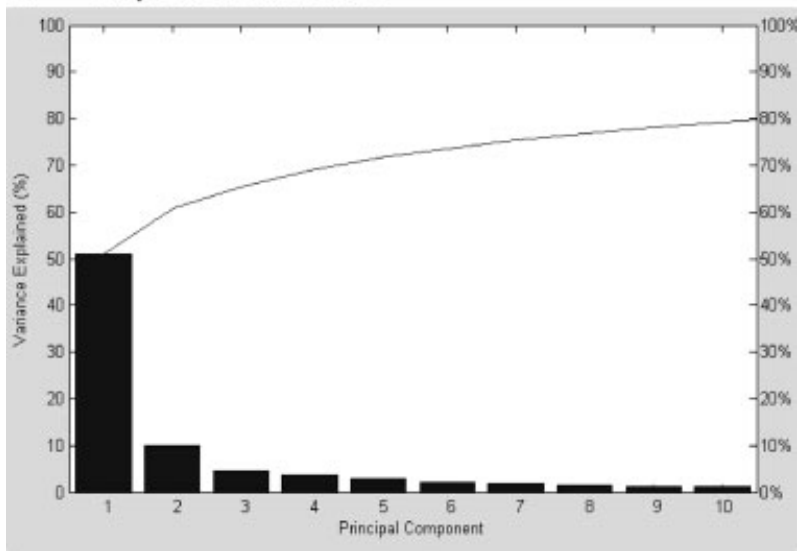

Figure 8. Supervised PCA result graphs. (A) The clustering of spectra acquired in three citologically different regions (see Fig. 7B) projected on the PCs planes. (B) The explained variance for each resulting principal component (histogram) associated with its cumulative value (line). The first PC explains more than $50 \%$ of the total variance. breast cancer clinical samples we developed a protocol using HIAR EDTA unlocking treatment followed by "on-target" trypsin digestion. In this paper we demonstrate that it might enable a suitable recovery of peptides for MS analysis of FFPE specimens.

Formaldehyde, contained in formalin solution, causes proteins to crosslink in a meshwork, stabilizing the protein mass and preserving tissue morphology. It is a strong reducing agent which, however, fixes protein by an oxidative reaction, forming methylene bridges between free amino groups on different polypeptide chains. Formaldehyde does not follow any particular chemical reaction with carbohydrates. Generally, triglycerides also remain unfixed. Most lipids dissolve during treatment with alcohol, xylene, or other clearant, during paraffin processing, and are not detectable in finished sections. The protein part of lipoprotein may well be fixed, and these too may resist extraction by solvents. Formalin penetrates tissue fairly quickly but the chemical reaction involved is much slower [16].

The potential application of clinical proteomics investigation to FFPE tissue samples have been particularly inter- 


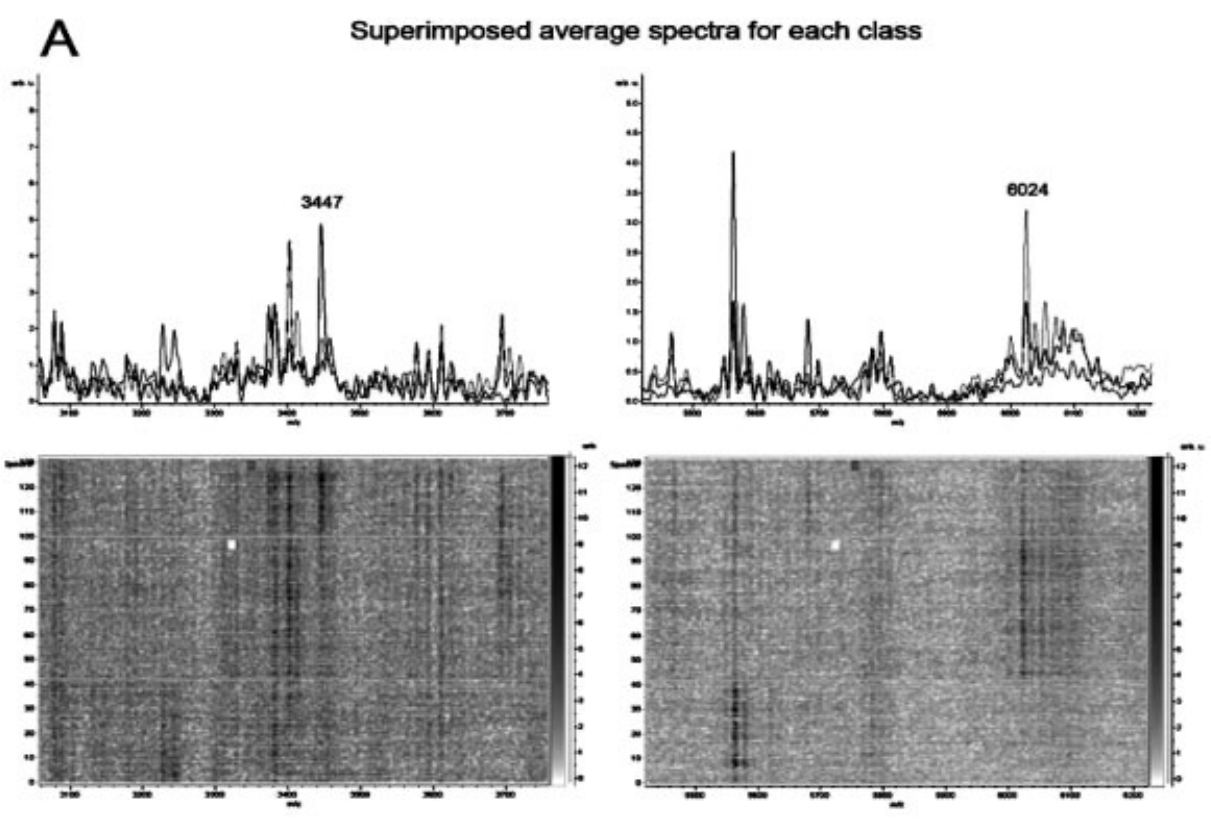

\section{B}

prediction on adipose tissue 2

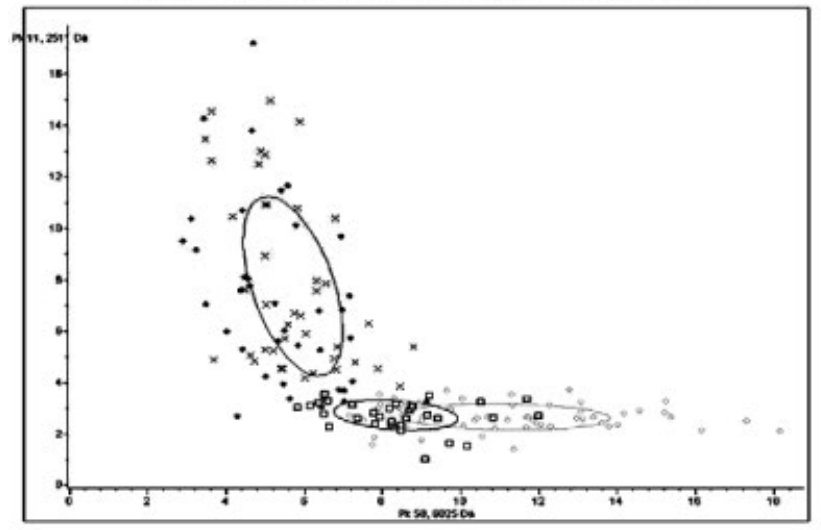

prediction on connective tissue
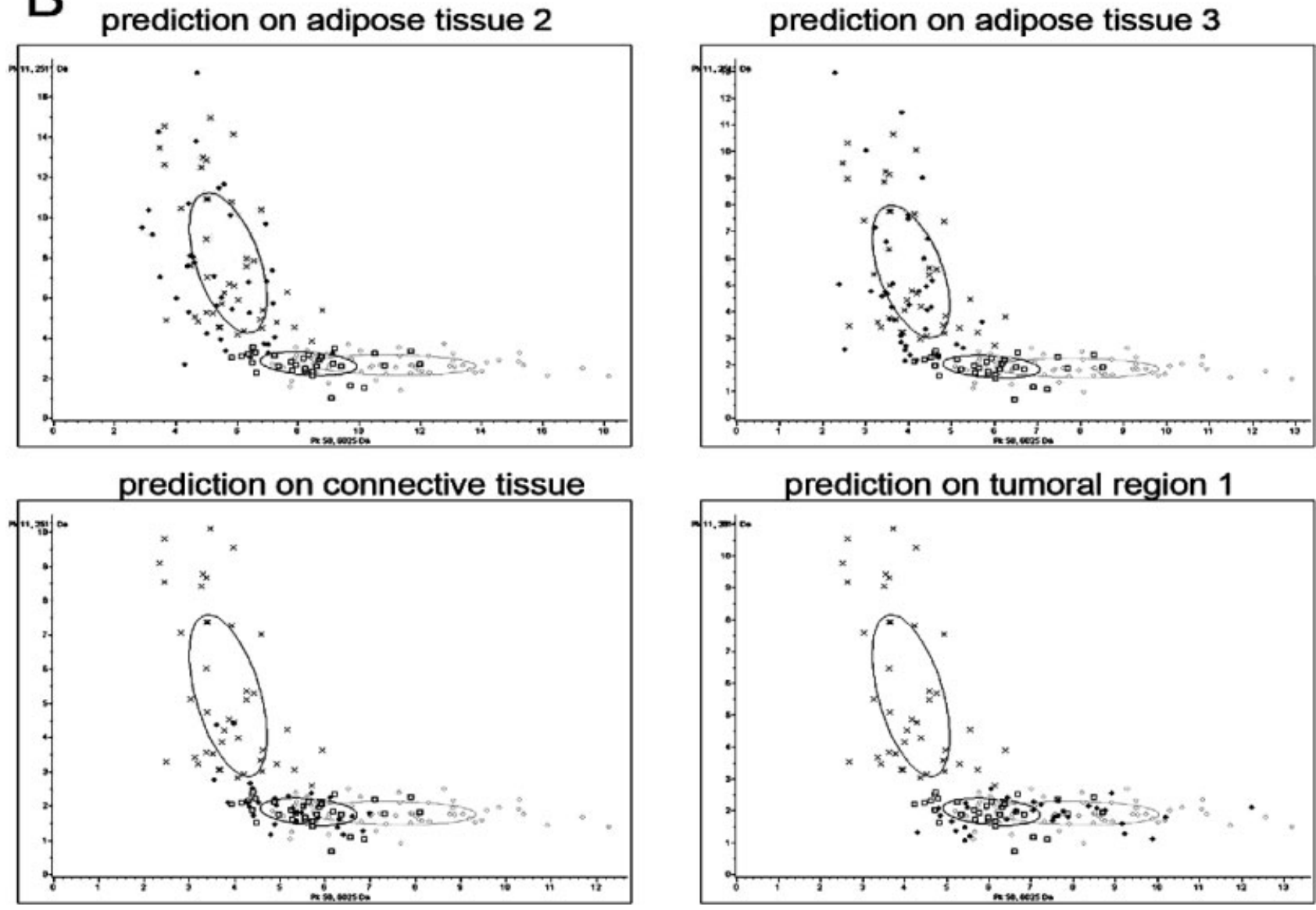

prediction on tumoral region 1

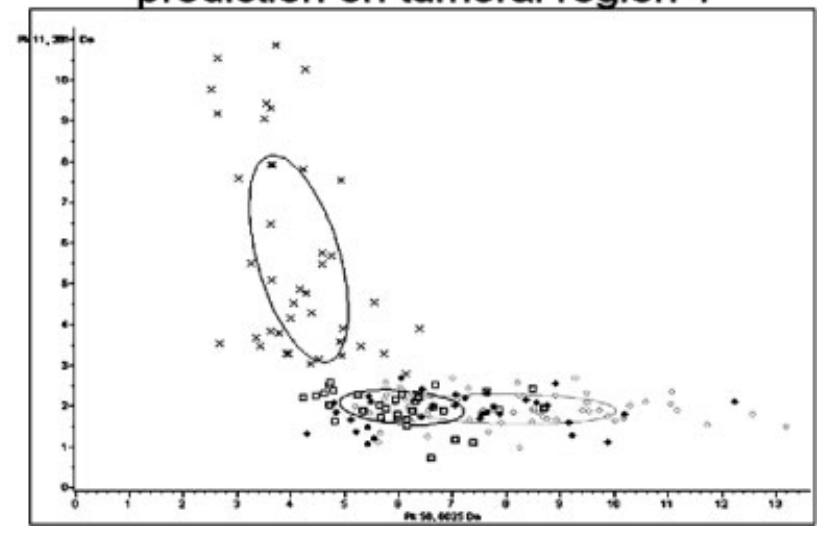

Figure 9. (A) Superimposed average spectra for the three class: cross = adipose tissue; square = connective tissue; diamond = tumoral region. Two of the signals with the highest variance inside the datasets are reported. (B) Graphical representation of the classification made on the basis of the intensity of the major differential signals on external datasets: black circles represent the predictions for the different regions. 
esting in the latest years due to the possibility open in the biomarker discovery effort by directly employing archive samples of biopsies. In this regard, the pioneering work of Hood et al. [17] has clearly demonstrated the possibility to apply a shotgun proteomics approach in FFPE protein profiling from clinical samples.

More recently, other studies have been pursuing the possible application of FFPE tissue analysis by MS and in particular MALDI-IMS. Lemaire et al. [13] showed that a combination of in situ extraction with in situ micro digestion performed on FFPE tissues allows the identification of proteins by nanoLC-nanoESI and MALDI imaging.

MALDI-TOF mass spectra quality for fresh frozen tissue is the best in terms of resolution, $\mathrm{S} / \mathrm{N}$ ratio, and number of signals. Nevertheless, the FFPE treatment is known to preserve the tissue morphology [16]. The strategy we developed to unlock protein peptides for MALDI-IMS analysis has taken advantage by the combination of heat induced antigen retrieval (HIAR) techniques. In fact, heating in various solutions has been reported to effectively increase immunostaining of many antigens in routinely processed FFPE material [18]. Several mechanisms have been proposed to explain HIAR such as the disruption of protein-protein crosslinks $[18,19]$ and/or of crosslinks involving calcium ions $[20,21]$ and the improvement of tissue permeability. The positive result of the EDTA treatment seems to agree with the controversial hypothesis on the role of calcium. In fact, after reacting with one amino group, formaldehyde can polymerize to form polioxomethylene chains. Calcium ions are able to coordinate to oxygen atoms in this polymer (bonds between the methyl groups of proteins introduced by formaldehyde treatment) and form a cage-like structure which prevent antigenantibody interactions [20, 21]. Nevertheless, the effect of calcium ions on immunoreactions might be selective for certain tissue types and not a universal phenomenon [16].

Many investigators have applied proteolytic digestion to FFPE specimens demonstrating its usefulness to restore immunoreactivity with a high $\mathrm{S} / \mathrm{N}$ ratio $[16,22,23]$. Proteolytic digestion, at low temperature, does not usually dramatically intensify immunoreactions compared with heat treatment; however, the choice of specific proteolytic enzymes, such as trypsin, is particularly convenient for MS analysis $[14,17]$. In order to avoid the tryptic peptide diffusion we have employed a double step procedure. In the first passage we took advantage of the HIAR and EDTA treatment in order to expose protein charged residues thus preventing random entangling of polypeptides. Therefore, the second treatment with trypsin results to be particularly effective given expected solvent distribution of arginines and lysines.

Protein signal localization is still preserved in such a tissue preparation. In fact, our data highlight either specific signal distributions or multivariate unsupervised model, colored according to the histological areas as defined by the H\&E staining.
This study may provide an additional tool toward the application of MALDI-IMS in the clinical proteomics investigation enabling the use of FFPE histological samples to perform global protein expression profiling. Given the large retrospective collection of tissue stored in FFPE such a technological breakthrough might return a significant improvement to foster clinical biomarker research.

The authors have declared no conflict of interest.

\section{References}

[1] Fox, C. H., Johnson, F. B., Whiting, J., Roller, R. P. P., Formaldehyde fixation. J. Histochem. Cytochem. 1985, 33, 845853.

[2] Shi, S.-R., Gu, J., Turrens, J., in: Shi, S. -R., Gu, J., Taylor, C. (Eds.), Antigen Retrieval Techniques: Immunohistochemistry and Molecular Morphology, Eaton Publishing, Natick, MA 2000, pp. 17-40.

[3] Ahram, M., Flaig, M. J., Gillespie, J. W., Duray, P. H. et al., Evaluation of ethanol-fixed, paraffin-embedded tissues for proteomic applications. Proteomics 2003, 3, 413-421.

[4] Shi, S.-R., Liu, C., Balgley, B. M., Lee, C., Taylor, C. R., Protein extraction from formalin-fixed, paraffin-embedded tissue sections: Quality evaluation by mass spectrometry. J. Histochem. Cytochem. 2006, 54, 739-743.

[5] Prieto, D. A., Hood, B. L., Darfler, M. M., Guiel, T. G. et al., Liquid tissue: Proteomic profiling of formalin-fixed tissues. Biotechniques 2005, 38, 32-35.

[6] Crockett, D. K., Lin, Z., Vaughn, C. P., Lim, M. S., ElenitobaJohnson, K. S. J., Identification of proteins from formalinfixed paraffin-embedded cells by LC-MS/MS. Lab. Invest. 2005, 85, 1405-1415.

[7] Caprioli, R. M., Farmer, T. B., Gile, J., Molecular imaging of biological samples: Localization of peptides and proteins using MALDI-TOF MS. Anal. Chem. 1997, 69, 4751-60.

[8] Chaurand, P., Caprioli, R. M., Direct profiling and imaging of peptides and proteins from mammalian cells and tissue sections by mass spectrometry. Electrophoresis 2002, 23, 3125-3135.

[9] Schwartz, S. A., Reyzer, M. L., Caprioli, M. R., Direct tissue analysis using matrix-assisted laser desorption/ionization mass spectrometry: Practical aspects of sample preparation. J. Mass Spectrom. 2003, 38, 699-708.

[10] Bruker-Daltonics Technical Note 012, Innovative smartbeam laser technology enhances MALDI-TOF based proteomics applications, 2005.

[11] Maddalo, G., Petrucci, F., lezzi, E., Pannellini, T. et al., Analytical assessment of MALDI-TOF imaging mass spectrometry on thin histological samples. An insight in proteome investigation. Clin. Chim. Acta 2005, 35, 210-218.

[12] Schwartz, S. A., Weil, R. J., Thompson, R. C., Shyr, Y. et al., Proteomic-based prognosis of brain tumor patients using direct-tissue matrix-assisted laser desorption ionization mass spectrometry. Cancer Res. 2005, 65, 7674-7681.

[13] Lemaire, R., Desmons, A., Tabet, J. C., Day, R., Salzet, M. et al., Direct analysis and MALDI imaging of formalin-fixed, paraffin-embedded tissue sections. J. Proteome Res. 2007, 6, 1295-1305. 
[14] Fowler, C. B., Cunningham, R. E., O'Leary, T. J., Mason, J. T., 'Tissue surrogates' as a model for archival formalin-fixed paraffin-embedded tissues. Lab. Invest. 2007, 87, 836-846.

[15] Hood, B. L., Conrads, T. P., Veenstra, T. D., Mass spectrometric analysis of formalin-fixed paraffin-embedded tissue: Unlocking the proteome within. Proteomics 2006, 6, 41064114.

[16] Yamashita, S., Heat-induced antigen retrieval: mechanisms and application to histochemistry. Prog. Histochem. Cytochem. 2007, 41, 141-200.

[17] Hood, B. L., Darfler, M. M., Guiel, T. G., Furusato, B. et al., Proteomic analysis of formalin-fixed prostate cancer tissue. Mol. Cell. Proteomics 2005, 4, 1741-1753.

[18] Shi, S. R., Key, M. E., Kalra, K. L., Antigen retrieval in formalin-fixed, paraffin-embedded tissues: An enhancement method for immunohistochemical staining based on microwave oven heating of tissue sections. J Histochem. Cytochem. 1991, 39, 741-748.
[19] Werner, M., Von Wasielewski, R., Komminoth, P., Antigen retrieval, signal amplification, and intensification in immunohistochemistry. Histochem. Cell Biol. 1996, 105, 253-260.

[20] Morgan, J. M., Navabi, H., Schmid, K. W., Jasani, B., Possible role of tissue-bound calcium ions in citrate-mediated high-temperature antigen retrieval. J. Pathol. 1994, 174, 301-307.

[21] Morgan, J. M., Navabi, H., Jasani, B., Role of calcium chelation in high-temperature antigen retrieval at different $\mathrm{pH}$ values. J. Pathol. 1997, 182, 233-237.

[22] Huang, S. N., Minassian, H., More, J. D., Application of immunofluorescent staining on paraffin sections improved by trypsin digestion. Lab. Invest. 1976, 35, 383-390.

[23] Curran, R. C., Gregory, J., Demonstration of immunoglobulin in cryostat and paraffin sections of human tonsil by immunofluorescence and immunoperoxidase techniques. Effects of processing on immunohistochemical performance of tissues and on the use of proteolytic enzymes to unmask antigens in sections. J. Clin. Pathol. 1978, 31, 974983. 